\title{
Simulation of freight train operations with departures ahead of schedule
}

\author{
A. Lindfeldt \& H. Sipilä \\ Department of Transport Science, Royal Institute of Technology (KTH), \\ Sweden
}

\begin{abstract}
Many lines in Sweden have mixed traffic, with both passenger and freight trains. In contrast to passenger trains, freight trains can deviate significantly from their timetable. This study evaluates the effect of modelling freight trains running ahead as well as behind schedule. In previous Swedish studies freight trains have been modelled as being on time or delayed. RailSys is used to simulate a section of the Southern Main Line and a fictive double-track line. Simulation results are compared to data from real train operations. Results show that freight trains can be modelled in a more realistic way. This can improve simulation analysis of freight operations.
\end{abstract}

Keywords: train traffic, simulation, timetable, delay, freight trains, double-track.

\section{Introduction}

Simulation offers a powerful tool for evaluation of rail traffic performance and can be used to analyse effects of infrastructure modifications, timetable strategies, vehicle performance, delay development etc. In this paper simulation software RailSys is used. Operating processes are created on an event driven basis, so-called synchronous simulation $[1,2]$.

Two different modelling approaches concerning freight train operation are investigated. Simulations are made on both a real network and on a fictive line. A $400 \mathrm{~km}$ double-track section of the Southern Main Line (Katrineholm to Hässleholm), with mixed passenger and freight operations, is simulated including additional in- and outflowing traffic from other lines. The fictive case can be seen as one direction of a double-track line in which timetables and delay levels are varied. 


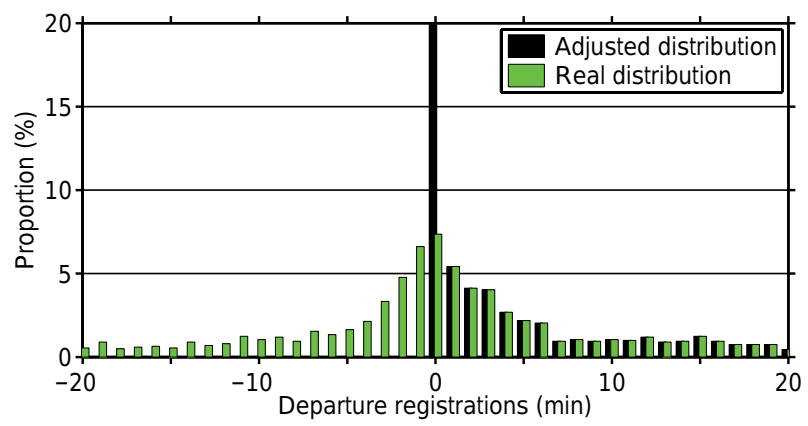

Figure 1: Example of freight train delay distribution. Positive values indicate delays. Zero level is $45 \%$ for the adjusted distribution.

All trains in Sweden have a specified schedule, this holds for regularly running trains as well as ad-hoc trains with a short planning horizon. Passenger trains follow their schedules reasonably well compared to freight trains and rarely run ahead of schedule. Freight trains however, show large deviations and can run ahead as well as behind their schedule. In previous studies done with RailSys in Sweden, freight trains have been modelled either on time or delayed.

This paper presents an approach to capture the variance in freight train operations. Train initialisation is modelled with both positive and negative deviations, i.e. ahead and behind of schedule. Stops allowing higher prioritized trains to pass slower freight trains (overtakings) are also frequently changed or cancelled due to operational reasons. Two different ways of modelling these types of stops are simulated.

\section{Methodology}

Previous RailSys based simulation studies in Sweden have not captured the effect with trains generated ahead of schedule. One benefit obtained from the work discussed in [3] is the possibility of changing delay inputs relatively fast after which a new perturbation file is generated. The same principles are also used in this study. Fig. 1 illustrates the difference between real departure registrations and how it has previously been modelled in simulations, i.e. early registrations add to the proportion of trains generated as specified by schedule.

Train stops are both a source for delays but can also be used to reduce delays, i.e. they can be shorter or longer than scheduled. Modelling stops accurately in simulations is crucial. Most scheduled passenger train stops are at stations where passenger exchange occurs, departing ahead of schedule is therefore not possible. Many of the scheduled freight train stops are due to overtakings and crossings. In reality these types of stops are cancelled or moved to another station if it is preferred from an operational point of view. This type of scheduled stop is modelled in two different ways in the simulations and are denoted conditional and 
unconditional stops. Conditional stops are possible to cancel in the simulations whereas unconditional stops cannot be cancelled.

Fig. 2 shows realised stop time as a function of scheduled stop time. This is based on empirical data for two months from several stations in the Swedish railway network [4]. Median as well as upper and lower quartiles are shown. Most passenger trains obey scheduled stop times quite well, freight trains on the other hand do not. In particular many of the longer scheduled stops take considerably shorter time than planned or are cancelled. Short stopping times do not only originate from late trains reducing delays, but also from trains departing ahead of schedule. The spread of realised stop times illustrate one of the reasons freight train stops are complex to model.
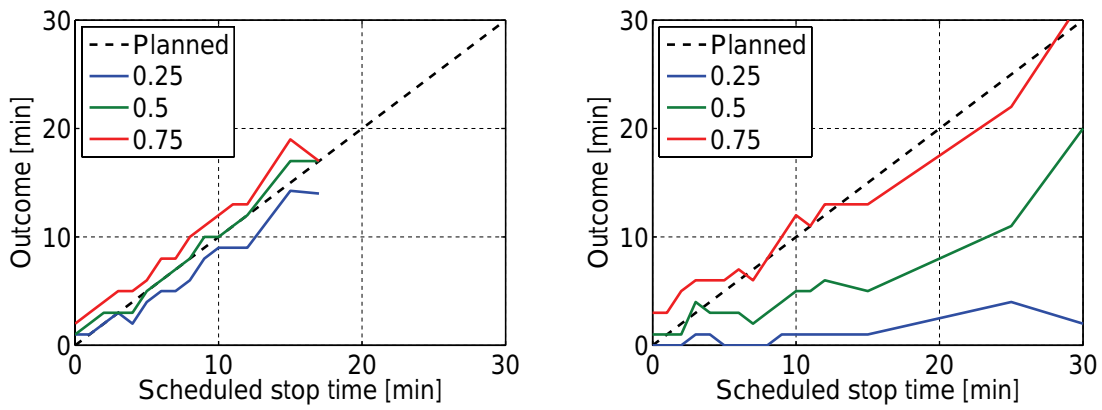

Figure 2: Realised stop time as a function of scheduled stop time. The data originates from stations located on both single and double-track lines in the Swedish railway network. Left: passenger trains. Right: freight trains.

\subsection{Effects of cancelled freight train stops on a fictive line}

Two cases are simulated to determine the effect of freight trains being allowed to cancel stops. The simulations are a subset of the factorial experiment reported in [5], where traffic mix and density, inter-station distance and primary delay levels are varied. The aim is to explain the creation of secondary delays and how timetable allowance is used to recover delays on a double-track line. Compared to the original setup, only timetables that include freight trains are used. Also, the inter-station distance is not varied in this study.

Fig. 3 shows the workflow, the simulation is repeated two times. In the first simulation freight trains use unconditional stops and cannot depart ahead of schedule. The second simulation uses conditional stops, i.e. departure ahead of schedule is possible. All other settings are unchanged.

The infrastructure model consists of one track operated in one direction. This represents a double-track where the two tracks are operated independently. The 


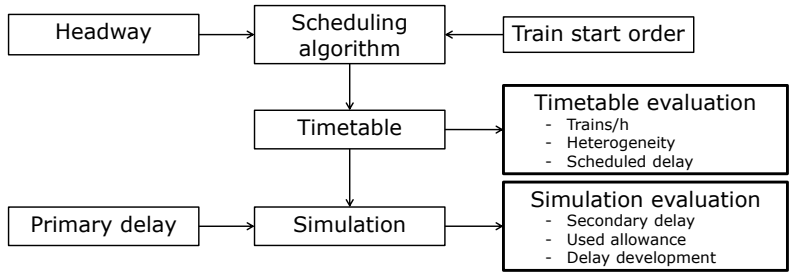

Figure 3: Simulation setup workflow.

line has overtaking stations with one side track spaced equidistantly at an interval of $20 \mathrm{~km}$. The total length of the simulated line is $200 \mathrm{~km}$.

The timetables are cyclic with up to three trains per cycle and include highspeed, InterCity and freight trains, see fig. 4. In the scheduling algorithm, timetables are controlled by starting order and headway of the trains. Faster trains are given priority over slower trains when overtakings are scheduled. One timetable consists of 35 consecutive cycles.

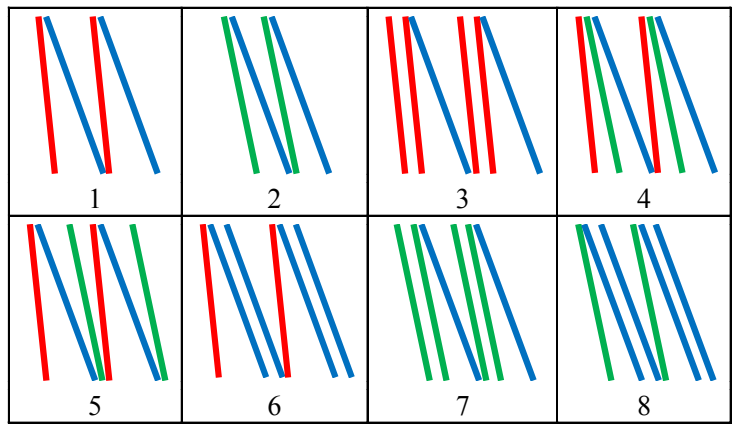

Figure 4: Timetable types (traffic mixes) used in simulations. High-speed (red), IC (green) and freight train (blue).

Perturbations include three different types of primary delays: initial delay, running time and dwell time extensions. All three types are varied coherently for two levels and are based on distributions compiled from empirical data defined in an earlier study [6]. All train types are assigned delays using the same stochastic distributions and no trains depart ahead of schedule at the origin station.

\subsection{Initialising freight trains ahead of schedule}

A primary (exogenous) delay or perturbation results normally from an incident that directly delays the train concerned. Examples of this are infrastructure or vehicle related problems, passenger exchange times etc. Interaction between trains can 
result in knock-on (secondary/reactive) delays. Whether a primary delay will result in knock-on delays or not and how large the impact will be depends on timetable allowances, buffer times, traffic density and so on.

An approach for modelling primary running time extensions is presented in [3]. Base distributions representing deviations from scheduled running times contain both primary and knock-on delays. These distributions are reduced in steps with the purpose of finding a level for the primary delays. The importance of including this type of perturbations is discussed in $[6,7]$. Registrations are also used for modelling initial delays (entry delays), i.e. trains entering a border or originating inside the simulated network. Fig. 5 describes the basic steps and workflow used for this part of the study, infrastructure is defined before this process. Variance in passenger train station stops is achieved by using dwell time extensions, these are based on manual measurements [6].

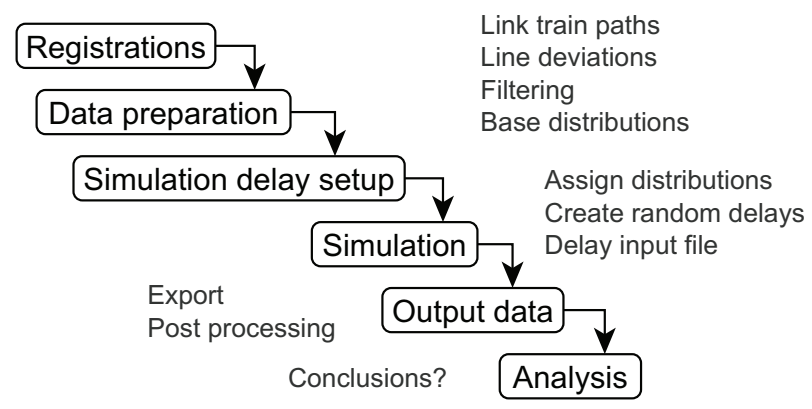

Figure 5: Workflow used in simulation study.

In order to use the real distributions, simulated freight trains get a constant deviation from their actual schedule. Initial delay distributions for different stations and train groups are defined relative this deviation, 60 minutes is used in this study. Evaluations are done with respect to the real schedule. Depending on the entry distributions used, this approach implies that almost every freight train will have a significant delay in the simulations which means that dispatching priorities need adjustments as well.

In the first scenario freight trains can be on time or delayed, stops cannot be cancelled (unconditional stop), but delay reduction is possible by minimising or reducing the scheduled stopping time. This can happen if there is no need for overtaking by a higher prioritised train. However, the train must still brake to stop and accelerate. The second scenario, in which freight trains can be initialised ahead of schedule, uses conditional stops.

Simulations model a normal weekday with both day and night operations, cycle time is 32 hours. Trains are generated outside the evaluated area (except in Katrineholm) which mean that they run a certain distance (4-30 km) depending on origin station used in the simulated timetable. This together with the cycle time give a warm-up period. 


\section{Results}

\subsection{Effects of cancelled freight train stops on a fictive line}

Overtakings occur on double-tracks supporting mixed traffic. Overtakings imply scheduled delay for the slower trains as they are required to stand aside and let faster trains pass. Also, overtakings introduce dependencies between trains that may cause delay transfer. Greater speed differences and traffic densities mean more frequent overtakings. Fig. 6 shows distributions of the realised running times for the train types in timetable type 4 (fig. 4), at three different traffic densities. Simulation results for the case with unconditional stops are indicated in darker colours and for conditional stops in lighter colours. Scheduled running times are marked by triangles.

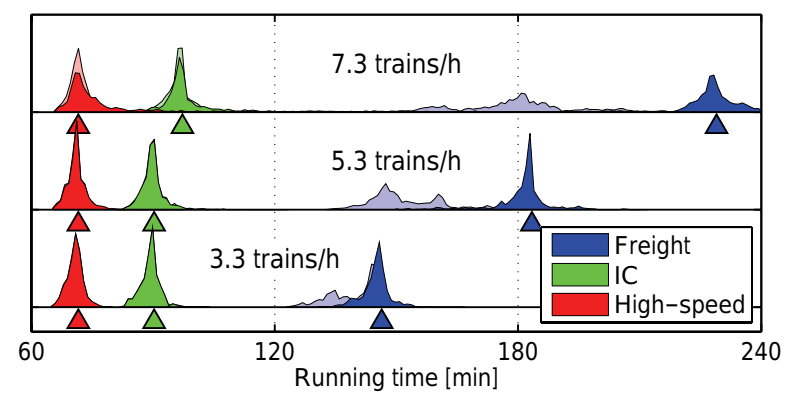

Figure 6: Results for timetable type 4, high primary delay level.

The increase in scheduled running time with higher traffic densities is not so large for IC-trains but more severe for freight trains, the slowest train type. In the case with unconditional stops, running time distributions are centered on the scheduled running times for all train types. The realised running time can be shorter than scheduled when late trains use available running time allowance and allowance at stops to reduce delays. Longer running times are due to primary and secondary delays.

The effect of allowing freight trains to cancel stops and depart ahead of schedule is most apparent in realised running times for the freight trains. High-speed and IC-train distributions remain almost unchanged. It is apparent that many freight trains, when allowed to cancel stops, can reduce their running times without delaying other train types significantly. The explanation is that they are not waiting unnecessarily for scheduled overtakings that will not occur due to delays. The effect is more dominant at higher traffic densities, due to more frequent overtakings. However, the time gain varies clearly.

Fig. 7 shows mean and standard deviation of running times for freight and high-speed trains, where values from the simulation using conditional stops have been normalised by corresponding values from the simulation of unconditional 
stops. Values larger than one indicate an increase and values smaller than one a decrease when conditional stops have been used. At higher traffic densities, all timetables show a decrease in mean value of running time and an increase in standard deviation. The reduction in mean delay correlates to the total time freight trains are scheduled to stand on side tracks and the number of overtakings that are realised in the simulation.

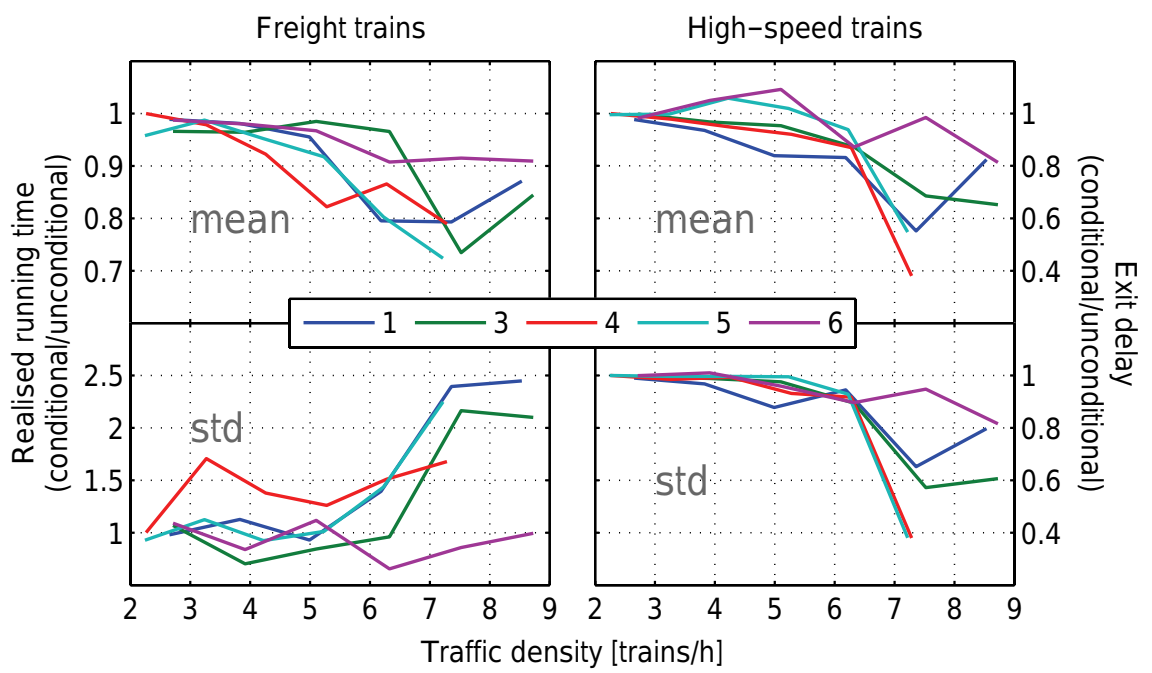

Figure 7: Results for freight and passenger trains. High level of primary delays.

Passenger train performance is evaluated by mean and standard deviation of their delay at the destination, i.e. exit delay. Results for IC-trains are similar with high-speed trains. Both mean and standard deviation decrease at the highest traffic densities and is explained by that the number of larger delays (>30 min) is reduced. At very high capacity utilisation, overtakings are scheduled at almost every station and the capacity of the stations is used to the maximum. This limits the possibilities to move overtakings in time or to another location when delays occur.

When freight train stops can be cancelled, they can adapt to late passenger trains and use capacity when it is available. This in turn frees up capacity at stations that is possible to use for unscheduled overtakings. The result may however depend on that capacity of the line is fairly constant with no severe bottlenecks downstream, in which case it might be unfavourable if too many trains arrive ahead of schedule.

Fig. 7 show results for simulations applying large primary delays. When smaller primary delays are used, the results remain approximately same for freight trains while the positive effect on passenger train delays is no longer evident. It is reasonable that the effect of increased capacity at stations for unscheduled 
overtakings is smaller when trains are operated closer to the scheduled timetable and the flow of trains is more even.

\subsection{Initialising freight trains ahead of schedule}

Evaluation is done by comparing simulated and observed outcome. The modelled and real timetable are similar but not equal, differences concern mostly freight trains. Observed outcome is based on around 100 days of operation. Different measures are available for estimating operational outcome of a rail network, e.g. on-time performance, mean values, timetable stability etc. In this study and also in the preceding work mean and standard deviation is used to describe outcome.

Fig. 8 shows outcome for two cases with different initial freight train delay distributions. The figure covers southbound direction from Katrineholm (K) to Hässleholm (Hm) with some larger intermediate stations. Left diagram shows the case with unconditional stops and adjusted initial distributions, right diagram is with conditional stops and real initial distributions (fig. 1). The highest frequency of freight trains is on the section between Mjölby (My) and Hässleholm. Only long distance freight trains are evaluated, however there is also a large amount of freight trains running shorter distances on the studied network.
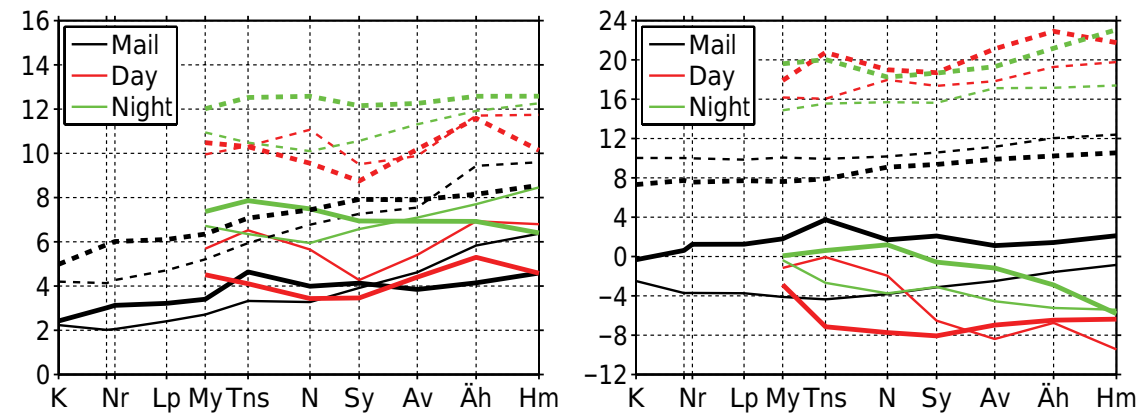

Figure 8: Simulated (thin) and observed outcome (bold) for southbound freight train groups. Mean (solid) and standard deviation (dashed) in minutes.

As previously mentioned also the scheduled stops are handled differently. The data set used for observed outcome is equal in both scenarios, it is however calculated differently. All registrations ahead of schedule are treated as on time which gives higher mean and lower standard deviation compared to the second case. The purpose is to increase comparability with the simulated outcome. In both cases only values with maximal deviation \pm 60 minutes are considered.

When negative initial deviations are possible, i.e. ahead of the modelled schedule, mean values are mostly below zero and standard deviation increases. This can of course be expected by studying fig. 1 in which it is straightforward that mean is lower and standard deviation higher for the real distribution compared to 
the adjusted one. Even though that example represents one station and direction for a set of freight trains, it is quite representative for many of the stations where trains are initialised in the simulations.

It can also be of interest to study development along the line given an initial interval with respect to schedule deviation. Both for passenger and freight trains, this can be useful in finding possible thresholds over which the probability of recovering from an initial delay is small. For passenger trains, especially for highspeed trains, missing a scheduled train slot can cause significant delay increase if obstructed by slower trains.

Fig. 9 shows deviation development for northbound freight trains with the three groups used in fig. 8 combined. Three start deviation intervals are used in Hässleholm (Hm) and distributions are calculated for Nässjö (N) and Mjölby (My). Intervals used are -30 to $-10,-10$ to 10 and 10 to 30 minutes deviation from schedule.
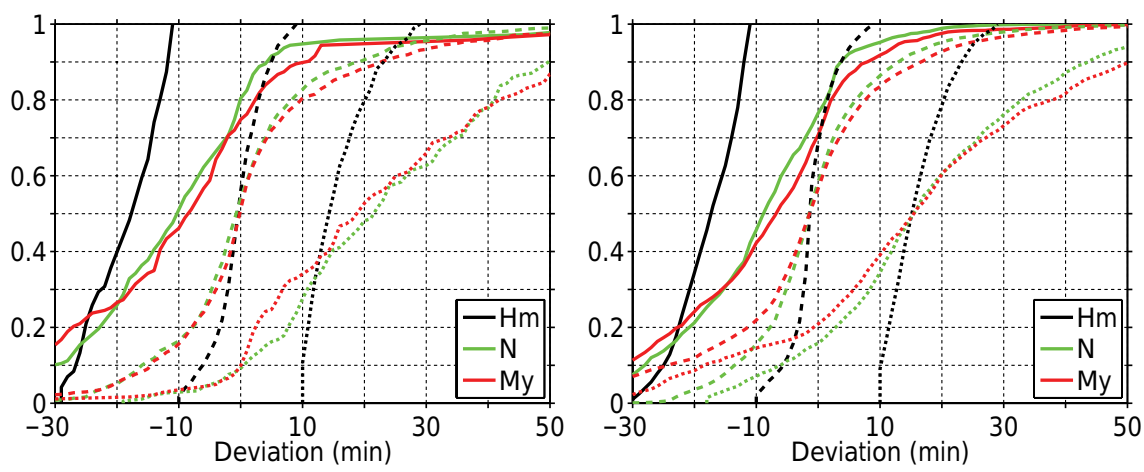

Figure 9: Distributions showing observed (left) and simulated (right) deviation development for northbound freight trains. Start intervals are defined by distribution in Hässleholm (Hm). Positive values indicate delays.

Around $75 \%$ of the total amount of train runs are located in the middle interval, i.e. \pm 10 minutes deviation. The remaining observations are split approximately evenly in the other two groups. From fig. 9 it can be observed that of the trains located in the middle interval, around $60 \%$ remain at the last evaluated station. For the other two intervals corresponding proportion is around $30 \%$. Partly this can be explained by the official statement that trains on time should have priority over trains with higher schedule deviations. The fit between observed and simulated outcome is relatively good. Influence on passenger trains is low even though the deviation for freight trains is increased. This is in part explained by the higher dispatching priority given to the passenger train. 


\section{Conclusions}

Adjusting simulation timetable, stop types and initial delays make it possible to model trains running ahead of schedule in RailSys. Results show that freight trains can be modelled in a more realistic way. This can improve simulation analysis of freight operations. In previous simulation studies of mixed traffic in Sweden, the effect on passenger trains of not modelling early freight trains has been assumed to be small.

This study confirms that this is the case under most conditions. A positive effect on the delays, i.e. reduction of mean and standard deviation, is observed when the capacity utilisation is extremely high and the primary delays are high. For freight trains, being able to cancel stops and depart ahead of schedule gives a reduction in mean running time, but also a considerable increase in standard deviation.

Timetable allowance and cancelled or shortened scheduled stops, allow freight trains to recover delays. On the other hand, lower operational priority (introducing unscheduled stops), extended shunting and so on cause delays. The variance is high and some of the trains included in the evaluated groups have run a long distance before entering the modelled network. Dependencies between different trains at marshalling yards also adds to this high variance.

Trains close to be on time should also have a higher probability of actually carry out their scheduled stops, especially when mixed with passenger trains. Compared to freight trains they normally have significantly higher on-time performance levels. Being ahead of schedule can also impose problems considering driver relief times and shunting operations. Although these stops are modelled in the simulations, no further study has been made and no dwell perturbations are used for freight trains.

Considering the original simulation setup, with no possibility of early trains, the presented approach adds realism to the simulations by better capturing the variance in freight operations. This gives possibilities to do more detailed freight oriented studies on lines and networks. Many of the rail traffic simulation studies presented are focused on passenger train performance. However, it has to be emphasized that rail traffic can be very complex and a model will always have limitations. Obtaining better compliance with respect to reality can improve understanding and also the possibilities of drawing conclusions regarding the performance of rail networks.

\section{References}

[1] Radtke, A. \& Hauptmann, D., Automated planning of timetables in large railway networks using a microscopic data basis and railway simulation techniques. Computers in Railways IX, eds. J. Allan \& C.A. Brebbia, WIT Press, pp. 615-625, 2004.

[2] Radtke, A., Timetable management and operational simulation: methodology and perspectives. Computers in Railways X, eds. J. Allan \& C.A. Brebbia, WIT Press, pp. 579-589, 2006. 
[3] Sipilä, H., Calibration of simulation model on the souther main line in sweden. Railway Engineering 2011, ed. M. Forde, Engineering Technics Press, 2011.

[4] Lindfeldt, A., A study of the performance and utilization of the swedish railway network. First International Conference on Road and Rail Infrastructure - CETRA 2010, ed. S. Lakušić, Department of Transportation, Faculty of Civil Engineering, University of Zagreb, pp. 889-895, 2010.

[5] Lindfeldt, A., Investigating the impact of timetable properties on delay propagation on a double track line using extensive simulation. Railway Engineering 2011, ed. M. Forde, Engineering Technics Press, 2011.

[6] Nelldal, B.L., Lindfeldt, O., Sipilä, H. \& Wolfmaier, J., Förbättrad punktlighet på X2000 - Analys med hjälp av simulering (in Swedish). Technical Report TRITA-TEC-RR 08-001, KTH, 2008.

[7] Lindfeldt, O. \& Sipilä, H., Validation of a simulation model for mixed traffic on swedish double-track railway line. Railway Engineering 2009, ed. M. Forde, Engineering Technics Press, 2009. 